\title{
The flavonoid, naringenin, decreases adipose tissue mass and attenuates ovariectomy-associated metabolic disturbances in mice
}

\author{
Jia-Yu Ke ${ }^{1}$, Kara L Kliewer ${ }^{1}$, Essam M Hamad ${ }^{2}$, Rachel M Cole ${ }^{1}$, Kimerly A Powell ${ }^{3}$, Rebecca R Andridge ${ }^{4}$, \\ Shana R Straka ${ }^{5}$, Lisa D Yee ${ }^{5}$ and Martha A Belury ${ }^{1 *}$
}

\begin{abstract}
Objective: Adverse metabolic changes associated with loss of ovarian function increase the risk of developing metabolic syndrome and non-alcoholic fatty liver disease (NAFLD) in postmenopausal women. Naringenin improves metabolic disturbances in vitro and in vivo. In the present study, we tested the effects of naringenin on metabolic disturbances resulting from estrogen deficiency in ovariectomized mice.

Materials/methods: Ovariectomized C57BL/6 J female mice were fed a control diet (10\% calories from fat) for 11 weeks. Mice either continued on the control diet $(n=9)$ or were switched to the control diet supplemented with 3\% naringenin $(n=10)$ for the next 11 weeks. Energy expenditure was measured by indirect calorimetry and activity was monitored by infrared beam breaks. Intra-abdominal and subcutaneous adiposity was evaluated by magnetic resonance imaging (MRI). Blood biochemical measures of metabolic response included glucose, insulin, adipokines, and lipids. Lipid content in liver and muscle and expression of relevant genes in adipose tissue, liver, and muscle were quantified.

Results: Ovariectomized mice fed naringenin exhibited lower fasting glucose and insulin levels compared to controls, with over $50 \%$ reduction of intra-abdominal and subcutaneous adiposity. Plasma leptin and leptin mRNA in adipose depots were also decreased in mice fed a naringenin diet. Monocyte chemoattractant protein-1 (MCP1/CCl2) and interleukin 6 (IL-6/ll6) mRNA expression levels were significantly lower in perigonadal adipose tissue of naringenin-supplemented mice. We also observed that mice fed a naringenin diet had less hepatic lipid accumulation with corresponding alterations of hepatic gene expression associated with de novo lipogenesis, fatty acid oxidation, and gluconeogenesis.
\end{abstract}

Conclusion: Dietary naringenin attenuates many of the metabolic disturbances associated with ovariectomy in female mice.

Keywords: Naringenin, Menopause, Obesity, Insulin sensitivity, Adipose tissue inflammation, Fatty liver

\section{Introduction}

During menopause, many women experience weight gain and accumulation of body fat in the waist region [1]. These changes of body composition increase the risk of developing metabolic syndrome [2], non-alcoholic fatty liver disease [3], and heart disease [4]. Although exogenous estrogen has been shown to be protective against

\footnotetext{
* Correspondence: Belury.1@osu.edu

'Department of Human Sciences, Human Nutrition Program, College of Education and Human Ecology, The Ohio State University, Campbell Hall 302, 1787 Neil Avenue, Columbus, Ohio 43210, USA

Full list of author information is available at the end of the article
}

many menopause-related metabolic abnormalities, longterm usage of hormone replacement therapy may increase the risk of breast cancer in addition to negative implications for cardiovascular diseases in postmenopausal women [5]. Lifestyle changes to reduce body weight, including healthy diet and regular exercise, are the initial strategies recommended for prevention and treatment of menopause-related metabolic disturbances.

Biologically active phytochemicals have attracted considerable attention for their potential health-promoting benefits [6,7]. Naringenin is a flavonoid that is abundant in citrus fruits and tomatoes $[8,9]$. Previously, we found 
that naringenin acts in a manner similar to metformin, a medicine used for treating type 2 diabetes, to reduce hepatic glucose production in hepatocytes [10]. In addition, naringenin improved some aspects of glucose and lipid homeostasis and mitigated adipose tissue inflammation in vivo [10-15]. However, the effects of naringenin on adipose depot mass and metabolic abnormalities associated with estrogen deficiency have not been studied.

Metabolic changes induced by estrogen depletion from ovariectomy share many similar characteristics with changes in menopausal women that are independent of energy intake, e.g., weight gain, increased adiposity, adipose tissue inflammation, and the development of fatty liver with inflammation $[16,17]$. These similarities make ovariectomized mice a good model to study physiological changes after menopause. Therefore, in the present study we investigated the effect of $3 \% \mathrm{wt} / \mathrm{wt}$ naringenin supplementation in female ovariectomized (OVX) mice. The aim of our study is to determine whether naringenin ameliorates weight gain and attenuates accumulation of subcutaneous and abdominal adipose tissues, with resultant decreases in fasting glucose and ectopic lipid accumulation in muscle and liver of OVX mice. Our findings suggest that many of the effects of naringenin on dysregulated metabolism are related to effects of reduced adipose mass and ectopic lipid deposition in muscle and liver of OVX mice fed diet with naringenin.

\section{Materials and methods Animals and diets}

Twenty-week old C57BL/6 J female mice were ovariectomized at 19 weeks old (Jackson Laboratory; Bar Harbor, ME, USA) and housed 4-5 per cage at $22 \pm 0.5^{\circ} \mathrm{C}$ on a 12:12-h light-dark cycle. After 2 weeks of acclimation, mice $(n=19)$ were fed ad libitum a semi-purified diet for 11 weeks to enhance weight gain (D12450J, Research Diets Inc. New Brunswick, NJ, USA, formula is shown in Additional file 1: Table S1). Then mice were randomized by weight into either CON $(n=9)$ or NAR $(n=10)$ group (randomization was based on 19 mice as one mouse died prior to assignment to diet groups). For the next 11 weeks, the CON group continued on the control diet and the NAR group received the control diet supplemented with 3\% wt/wt naringenin (Sigma, St. Louis, MO, USA), custom prepared by Research Diets Inc. (formula is shown in Additional file 1: Table S1). This dose of naringenin is based on a previously published study showing amelioration of metabolic disturbances in C57BL/6 J male mice fed a high-fat diet with $3 \%$ naringenin [12]. Body weight and food intake were measured daily. At week 22, mice were fasted for $5 \mathrm{~h}$, anesthetized with isoflurane for blood collection via cardiac puncture, and then euthanized by cervical dislocation. Blood was collected into EDTAcoated blood collection tubes and plasma was obtained after centrifugation. Tissues, including subcutaneous adipose tissue (SCAT, thoracic and abdominal mammary fat pads), liver, perigonadal adipose tissue (PGAT), and quadriceps skeletal muscle, were excised, weighed, snap frozen in liquid nitrogen, and stored at $-80^{\circ} \mathrm{C}$ until further analysis. All procedures were in accordance with institution guidelines and approved by the Institutional Animal Care and Use Committee at The Ohio State University.

\section{Fasting glucose analysis}

At weeks 5 and 18, glucose was measured from tail vein blood samples after a $5 \mathrm{~h}$ period of fasting (OneTouch Ultra blood glucose meter, LifeScan Inc., Milpitas, CA, USA).

\section{Plasma analysis}

Plasma insulin, leptin, and adiponectin levels were measured by ELISA (Millipore, Billerica, MA, USA) according to the manufacturer's instructions. Plasma triglycerides were examined using a Cholestech LDX analyzer (Cholestech Corporation, Hayward, CA, USA). Plasma free fatty acids (NEFA C, Wako Chemicals, Richmond, VA, USA) and total cholesterol (Pointe Scientific Inc., Canton, MI, USA) were determined by enzymatic colorimetric assays.

\section{Indirect calorimetry}

During week 17, six mice in each group were housed individually in metabolic chambers at $22^{\circ} \mathrm{C}$, allowed free access to food and water, and acclimated 24 hours prior to metabolic assessments. Measurements were taken for a 24-h period, including a 12-h light cycle and a 12-h dark cycle. Oxygen consumption $\left(\mathrm{VO}_{2}\right)$, carbon dioxide production $\left(\mathrm{VCO}_{2}\right)$, and physical activity (by infrared beam breaks) were measured every 20 minutes using a computer-controlled, open-circuit Oxymax/CLAMS System (Columbus Instruments, Columbus, OH, USA). Respiratory exchange ratio (RER) was calculated as the ratio of $\mathrm{VCO}_{2}$ to $\mathrm{VO}_{2}$. Heat, the standard measure of energy expenditure, was calculated with the formula [18],

$$
\begin{gathered}
\text { Heat }(\mathrm{kcal} / \mathrm{hr})=(3.815+1.232 \times \mathrm{RER}) \times\left(\mathrm{VO}_{2}\right) \times \\
(1 \mathrm{~L} / 1000 \mathrm{ml} \times 1 \mathrm{~kg} / 1000 \mathrm{~g}) \times(\text { body weight })
\end{gathered}
$$

\section{Magnetic Resonance Imaging (MRI)}

During week 19, total, intra-abdominal, and subcutaneous adiposity were analyzed by MRI using a Bruker Biospin 94/30 magnet (Billerica, MA, USA) and a $70 \mathrm{~mm}$ diameter linear volume coil. T1-weighted coronal images of the whole mouse torso were collected using a respiratorygated RARE sequence $(\mathrm{TR} / \mathrm{TE}=1570 / 7.5 \mathrm{~ms}$, RARE factor $=4$, FOV $=70 \times 45 \mathrm{~mm}^{2}$, matrix size $=256 \times 192$, slice 
thickness $=1 \mathrm{~mm}$, navg = 2). The details and definition of fat areas are shown in Additional file 1: Figure S1.

\section{Histology}

Liver samples were collected at necropsy and fixed in $10 \%$ neutral buffered formalin overnight and transferred to $70 \%$ ethanol for storage. Tissues were then processed, embedded in paraffin, sectioned, and stained with hematoxylin and eosin (H\&E).

\section{Total lipids, triacylglyceride and diacylglyceride analysis} of liver and muscle

Total lipids were extracted from liver or muscle samples with 2:1 (v/v) chloroform: methanol and washed with 0.88\% KCL [19]. The chloroform phase was transferred to a weighed test tube and dried under nitrogen gas at room temperature. The test tube containing the dried sample was weighed again to calculate total extracted lipid. Following the lipid extraction, triacylglycerol and diacylglycerol were obtained using solid-phase extraction [20] and solubilized in tert-butanol, methanol, Triton $\mathrm{X}-100$ [21]. Analysis was performed using enzymatic colorimetric assay [22].

\section{RNA extraction and quantitative real-time polymerase chain reaction (qRT-PCR) analysis of gene expression}

Total RNA was extracted from liver and muscle samples with QIAzol lysis reagent (Qiagen, Valencia, CA, USA) and from perigonadal adipose tissue and subcutaneous adipose tissue using RNeasy Lipid Tissue Mini Kit (Qiagen) following manufacturer's instructions. Total RNA was then reversed transcribed to cDNA using High Capacity cDNA Reverse Transcription Kit (Applied Biosystems, Foster City, CA, USA). qRT-PCR analysis was performed with ABI Prism 7300 sequence detection system (Applied Biosystems) using TaqMan Gene Expression Assays (Applied Biosystems, Additional file 1: Table S2). Target gene expression was normalized to $18 \mathrm{~S}$ rRNA for perigonadal adipose tissue or glyceraldehyde 3-phosphate dehydrogenase (GAPDH) for liver, subcutaneous adipose tissue, and muscle. Endogenous Controls (VIC probes) were amplified in the same reaction and expressed as $2-\Delta \Delta C t$ compared to the CON group [23].

\section{Statistical analysis}

All data are presented as mean \pm standard error (SEM) with $\mathrm{p}<0.05$ considered significantly different. Statistical analysis was performed using SPSS version 20.0 software (SPSS, Inc., Chicago, IL, USA). Significance was determined using two-tailed unpaired Student's $t$ test. Caloric intake, heat, and adipose mass were also analyzed by ANCOVA with body weight as a covariate [24]. Pearson correlation coefficient was used for the correlation analyses.

\section{Results}

Effect of naringenin on caloric intake, body weight, and metabolic measurements

Caloric intake decreased significantly when mice were switched to a naringenin-containing diet at week 12 (Figure 1A) without complete recovery to baseline in the following weeks. However, excluding week 12, daily caloric intake from week $0-11$ to week 13-22 did not differ significantly between the two treatment groups $(\mathrm{p}=0.075)$. Body weights were significantly reduced in the NAR group from week 12 until the end of the study (Figure 1B). There were no differences in caloric intake between groups after adjusting for body weight (ANCOVA, $\mathrm{p}=0.508$ ). There were also no differences in ambulatory activity (Figure 1C) and energy expenditure after controlling for body weight differences between groups (Figure 1D).

Fasting glucose levels were significantly elevated in the CON mice from $121.8 \pm 5.8 \mathrm{mg} / \mathrm{dl}$ at week 5 to $163.0 \pm$ $5.2 \mathrm{mg} / \mathrm{dl}$ at week 18 (Figure $1 \mathrm{E}$ ), while unchanged in NAR mice $(128.6 \pm 4.8$ at week 5 and $127.1 \pm 10.2$ at week 18). Additionally, diets enriched with naringenin resulted in decreased fasting insulin levels and HOMA-IR values (Figure $1 \mathrm{~F} \& \mathrm{G}$ ), surrogate markers of insulin sensitivity. Using an insulin tolerance test, we observed that glucose levels were significantly lower in the NAR mice compared to the CON mice at $45 \mathrm{~min}(48.0 \pm 3.4 \mathrm{mg} / \mathrm{dl}$ vs. $64.8 \pm$ $8.1 \mathrm{mg} / \mathrm{dl})$ and $60 \mathrm{~min}(51.4 \pm 8.2 \mathrm{mg} / \mathrm{dl}$ vs. $77.0 \pm 6.1 \mathrm{mg} /$ dl) after injection of insulin (Additional file 1: Figure S2). However, no difference was observed in areas under the curve (AUC) between groups.

Effect of naringenin on adiposity, plasma adipokines, and adipose tissue gene expression

MRI analysis was performed in a subset of mice from both groups $(n=6)$, which revealed a reduction in total, intraabdominal and subcutaneous adiposity by 54,59 , and $50 \%$, respectively, in mice fed naringenin versus control diets (Figure 2A \& C). Additionally, naringenin was significantly associated with decreased perigonadal (PGAT) and subcutaneous (SCAT) adipose tissues (Table 1). Because body weights differed significantly between groups and body weight had a significant effect on perigonadal and subcutaneous adipose tissue mass $(\mathrm{p}<0.001)$, we used ANCOVA to adjust for this difference when comparing adipose tissue mass. Mice fed naringenin had significantly decreased perigonadal adipose tissue mass $(p=0.006)$ after controlling for body weight in comparison to control mice. Fasting plasma leptin levels decreased by $80 \%$ in the NAR mice (Figure 2B) and strongly correlated with total, intra-abdominal, and subcutaneous adiposity determined by MRI (total, $\mathrm{r}=0.85, \mathrm{p}=0.008$; intra-abdominal, $\mathrm{r}=0.86, \mathrm{p}=0.006$; subcutaneous, $\mathrm{r}=0.80, \mathrm{p}=0.016$ ), as well as both perigonadal and subcutaneous adipose tissue mass $(r=0.95$ and 0.97 respectively, both $\mathrm{p}<0.001)$. 


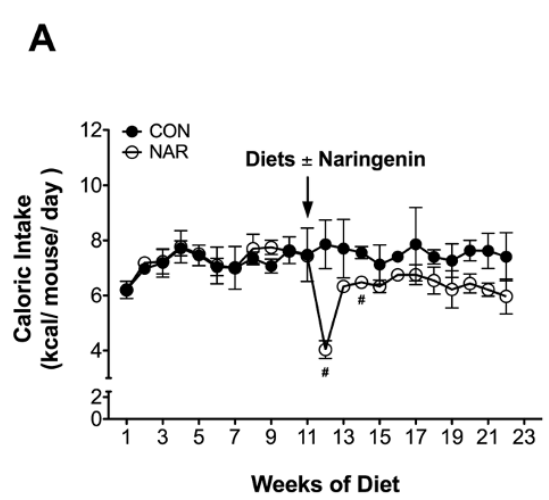

B

E

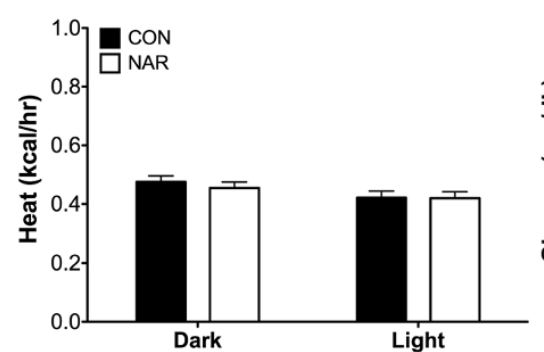

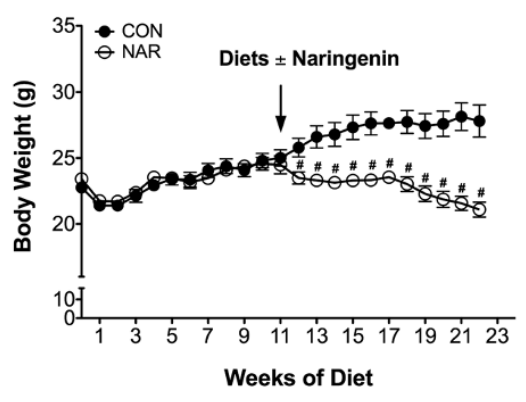

$\mathbf{F}$

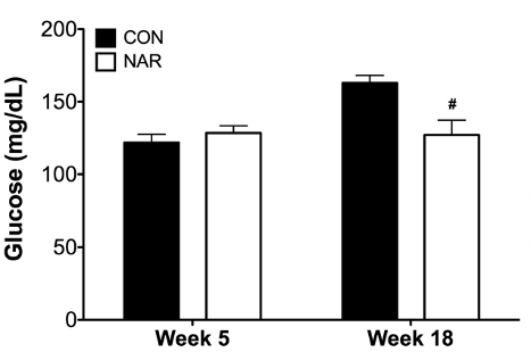

C

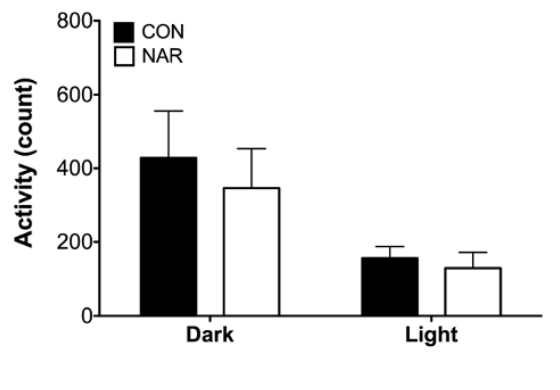

G

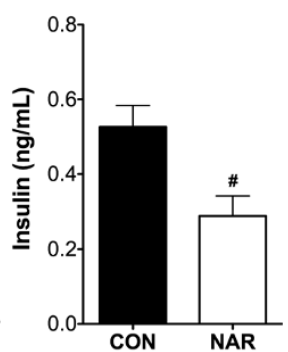

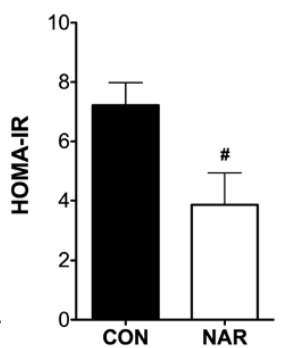

Figure 1 Effects of dietary naringenin on caloric intake, body weight, and metabolic measurements. OVX C57BL/6 J mice were fed the control diet for 11 weeks then randomized to continuation of the control diet $(n=9)$ or switched to $3 \% \mathrm{wt} / \mathrm{wt}$ naringenin supplementation of the control diet $(n=10)$ for the weeks 12-22. Average daily caloric intake (A) of two groups ( $n=2 /$ group) and weekly body weight (B) of the CON $(n=9)$ and NAR $(n=10)$ group were determined. At week 17, CLAMS chambers were used to measure locomotor activity (ambulation) in the horizontal plane by infrared beam breaks (C) and estimated energy expenditure (heat) after controlling for body weight (D) in the dark and light phase for 24-h following a 24-h acclimation ( $n=6 /$ group). Fasting glucose levels $(\mathbf{E})$ were measured at week 5 and week $18,(C O N, n=9$; $N A R, n=10)$. Fasting insulin levels $(\mathbf{F})$ were determined after 22 weeks of experimental period ( $n=6 /$ group). HOMA-IR values (G) were derived from fasting plasma glucose and insulin ( $n=4 /$ group). Values are presented as mean \pm SEM. Significance between groups was determined by Student's $t$ test, except metabolic data of heat was analyzed by ANCOVA with body weight as a covariate. ${ }^{\# P}<0.05$ compared CON with NAR.

Interestingly, plasma leptin levels correlated positively with insulin levels $(\mathrm{r}=0.76, \mathrm{p}=0.004)$, as previously shown in women [25]. Dietary naringenin was not associated with changes in levels of adiponectin in plasma and mRNA expression (Adipoq) in perigonadal adipose tissue between groups (Figure 2B \& D). Mice fed naringenin also had significantly lower leptin mRNA expression (Lep) in adipose depots, with a $60 \%$ reduction in perigonadal adipose tissue and a $55 \%$ reduction in subcutaneous adipose tissue (Figure 2D \& E).

We measured mRNA levels of genes encoding for several markers of inflammation related to obesity, including the chemokine MCP1 $(\mathrm{Ccl} 2)$, proinflammatory cytokine IL6 (Il6) and TNF $\alpha$ (Tnf), and macrophage-specific marker F4/80 (Emr1) in adipose depots (Figure 2D \& E). The diet with naringenin significantly down-regulated mRNA levels of MCP1 (56\% reduction) and IL6 (40\% reduction) in perigonadal adipose tissue, but had no significant effect on MCP1 and IL6 levels in subcutaneous adipose tissue. TNF $\alpha$ and F4/80 mRNA was not affected by the naringenin supplementation in perigonadal adipose tissue.

\section{Effect of naringenin on plasma, hepatic, and muscle lipid profile}

Dietary naringenin reduced total cholesterol levels (Figure 3A), but did not change the levels of triglyceride and non-esterified fatty acids in plasma (Figure 3B \& C). As ectopic lipid accumulation in liver has been observed in OVX animals [26], we tested hepatic lipid contents in both groups. Dietary naringenin decreased the extracted total lipids and triacylglyceride levels in the liver (Figure 3D \& E) but did not affect diacylglyceride levels (data not shown). Small lipid droplets were scattered throughout $H \& E$ stained liver sections from the CON group, while no lipid droplets were detected in the NAR group (Figure 3F). OVX has been shown to increase muscle lipid contents [27]. Therefore, we examined muscle lipid levels and found naringenin reduced the extracted total lipids levels (Figure 3G) but had no effect on either triacylglyceride 


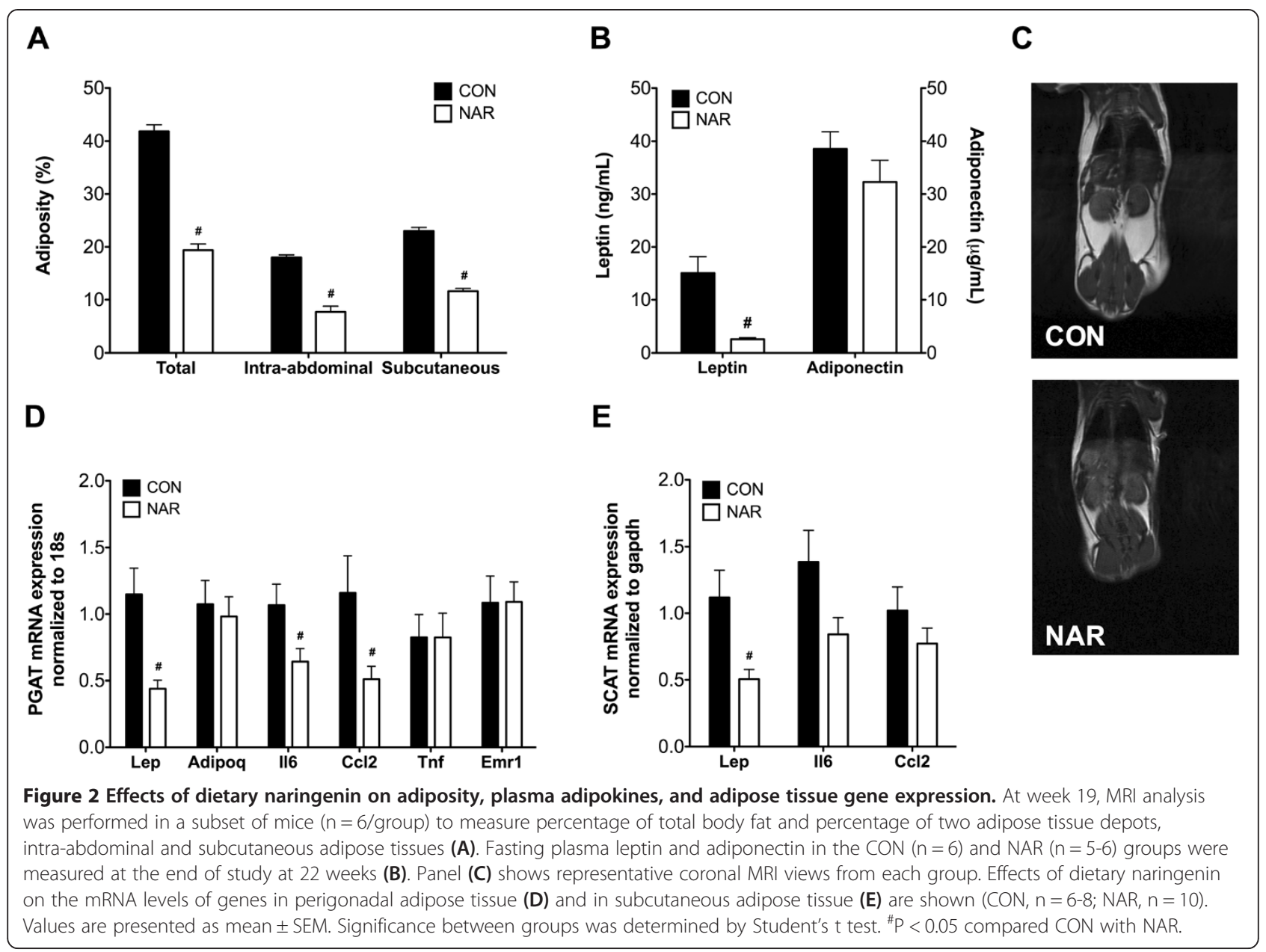

Table 1 Effect of dietary naringenin on tissue mass in OVX C57BL/6 J female mice

\begin{tabular}{ccc}
\hline & CON & NAR \\
\hline Tissue Weights (g) & & \\
Perigonadal Adipose Tissue & $1.44 \pm 0.15$ & $0.38 \pm 0.05^{\#}$ \\
Subcutaneous Adipose Tissue & $1.21 \pm 0.14$ & $0.52 \pm 0.03^{\#}$ \\
Liver & $0.94 \pm 0.05$ & $0.80 \pm 0.03^{\#}$ \\
Muscle (Quadriceps) & $0.36 \pm 0.01$ & $0.32 \pm 0.02$ \\
Tissue Weight Percentages (\%) & & \\
Perigonadal Adipose Tissue & $5.23 \pm 0.35$ & $1.81 \pm 0.19^{\#}$ \\
Subcutaneous Adipose Tissue & $4.34 \pm 0.34$ & $2.55 \pm 0.15^{\#}$ \\
Liver & $3.45 \pm 0.09$ & $3.89 \pm 0.12^{\#}$ \\
Muscle (Quadriceps) & $1.33 \pm 0.07$ & $1.55 \pm 0.07^{\#}$ \\
\hline
\end{tabular}

Significance between groups was determined by Student's t-test $(n=9-10)$. Data represent the mean \pm SEM.

\#P $<0.05$ compared NAR with CON.
(Figure $3 \mathrm{H}$ ) or diacylglyceride levels in skeletal muscle (data not shown).

\section{Effect of naringenin on hepatic and muscle mRNA expression}

Srebf1, Fasn, and Scd1 encode proteins involved in de novo lipogenesis. Increased hepatic expression of Srebf1 was observed in the NAR mice (Figure 4A). However, naringenin down-regulated Scd 1 mRNA by $38 \%$ but had no effect on another lipogenic enzyme, Fasn. No differences in mRNA levels of genes related to steatotic liver $[28,29]$ were detected, e.g., Pparg and Dgat2 (Figure 4A). Expression of genes involved in fatty acid oxidation, $\mathrm{Cpt} 1 \alpha$ (mitochondrial) was significantly higher in the mice fed a naringenin diet, but Acox1 (peroxisomal) was decreased (Figure 4B). PGC1 $\alpha$ (Ppargc1a) is a transcriptional coactivator regulating genes involved in fatty acid oxidation and gluconeogenesis. Dietary naringenin induced PGC1 $\alpha$ mRNA (4-fold) as well as PEPCK (Pck2) mRNA (3.5-fold), a rate-limiting enzyme in hepatic gluconeogenesis (Figure 4B), but not G6Pase (G6pc) mRNA, another enzyme involved in gluconeogenesis. Having observed 


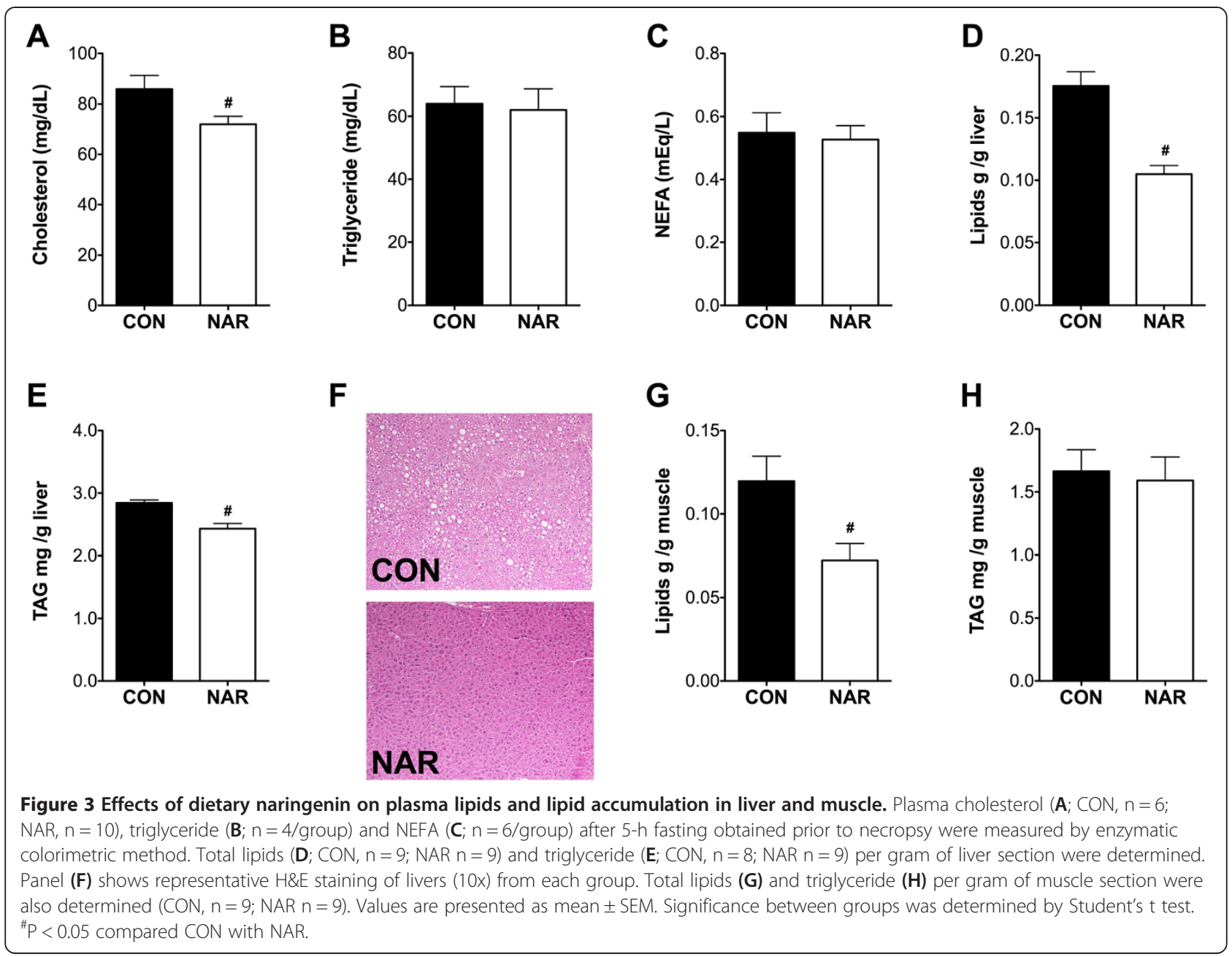

reduced lipid content in skeletal muscle, we measured the mRNA levels of genes involved in fatty acid metabolism (Figure 4C). However, naringenin had no effect on the expression of $\mathrm{Cpt} 1 \beta$ and PGC1 $\alpha$ in muscle. Interestingly, Fasn mRNA expression was higher in muscle tissue of NAR relative to $\mathrm{CON}$ mice but did not reach significance $(\mathrm{p}=0.07)$.

\section{Discussion}

Estrogen deficiency leads to metabolic changes and increased risk of metabolic syndrome [1-3,30]. To our knowledge, this is the first paper to examine the effects of naringenin on energy metabolism and adiposity in OVX female mice, a preclinical model of menopause. We report here that OVX mice exhibited metabolic disturbances expected with estrogen deficiency including elevated fasting glucose and obesity [3,26,31]. The addition of naringenin to the diet 1) decreased body weight, fasting glucose and insulin, 2) reduced body fat and perigonadal adipose tissue inflammation, 3) decreased plasma cholesterol and ectopic lipid accumulation in liver and muscle, and 4) affected expression of hepatic genes involved in de novo lipogenesis and fatty acid oxidation. Therefore, our study results suggest that a $3 \% \mathrm{wt} / \mathrm{wt}$ dietary naringenin supplementation ameliorates many metabolic derangements associated with estrogen deficiency.

We observed that naringenin supplementation caused an initial reduction of caloric intake, followed by a decrease in caloric intake and body weight over the subsequent ten weeks of feeding. Reduced caloric intake after naringenin supplementation in the present study was unexpected because previous studies had reported no differences in food intake in male rodent models supplemented with naringenin [11-13,32-34]. No changes in food intake were observed even in the same strain, wild-type C57BL/6 J males [12,35]. Discrepancies between our data from studies in C57BL/6 J males may be attributed to the fat content in the diet, age of the mice, and different effects of naringenin on appetite between male and female OVX mice.

Estrogen interacts with neuropeptides, decreases food intake, and reduces weight gain [36]. Hyperphagia has 


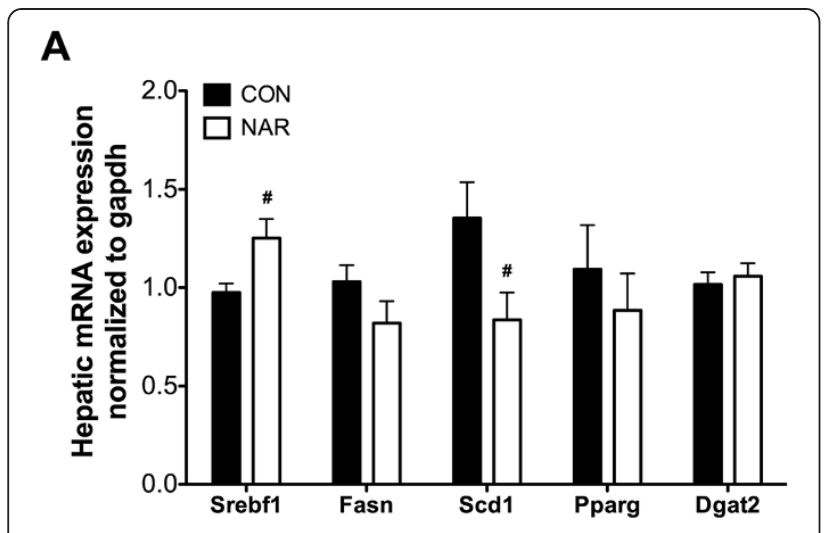

B

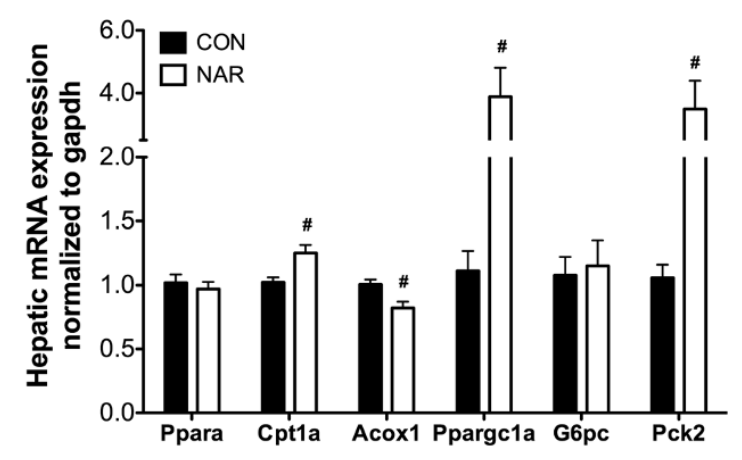

C

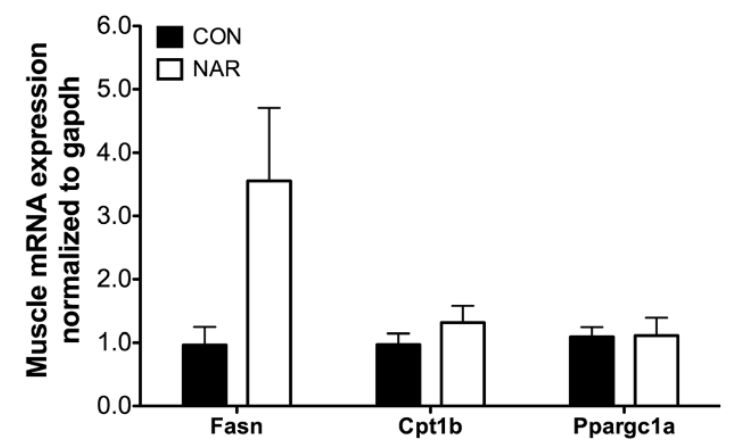

Figure 4 Effects of dietary naringenin on gene expression in liver and muscle. mRNA expression of hepatic genes related to de novo lipogenesis and hepatic steatosis (A), mRNA expression of hepatic genes related to beta-oxidation and gluconeogenesis (B), and mRNA expression of genes related to de novo lipogenesis and beta-oxidation in muscle (C) were quantified using qRT- PCR ( $n=6-10$ /group). Values are presented as mean \pm SEM. Significance between groups was determined by Student's $t$ test. ${ }^{\#} P<0.05$ compared CON with NAR.

been reported in some models of menopause [37] but not all [17,36-38]. Instead of altering food intake, several studies demonstrated that OVX mice have lower energy expenditure and activity levels, especially in the dark phase of the light/dark cycle [17,37-39]. These findings are consistent with human data demonstrating decreased free-living and $24 \mathrm{~h}$ energy expenditure, and reduced physical activity during the menopausal transition [40]. The totality of evidence suggests that reduced energy expenditure and increased energy efficiency, rather than overeating, results in the menopause-associated metabolic disturbances. We did not observe differences in spontaneous physical activity and in energy expenditure between groups after controlling for body weight. It is unclear whether the effects of naringenin on body weight and other OVX-related metabolic disturbances are attributed to reduced caloric intake and/or other mechanisms. In future studies, a pair-fed group will be included to control for possible differences in energy intake as a potential confounding factor.

Dietary naringenin attenuated hyperglycemia and hyperinsulinemia induced by a high-fat or a fructose-enriched diet $[12,33]$. We observed development of hyperglycemia in OVX mice from week 5 to week 18, consistent with Roger et al. who showed elevated fasting glucose in mice after 12 weeks of ovariectomy compared to sham-operated mice [16], while naringenin supplementation prevented the development of hyperglycemia and lowered fasting insulin concentration and HOMA-IR value. Potter et al. suggest that a significant difference in insulin resistance (determined by insulin tolerance test) may not develop until as late as 26 weeks post-ovariectomy [17], which may explain why insulin reduced blood glucose levels more effectively in mice supplemented with naringenin but the glucose AUC did not reach significance in the present study.

Estrogen deficiency increases the susceptibility to weight gain and central obesity in both humans and mice [11,16,41]. Hong et al. demonstrated that adiposity in OVX female mice, examined by DEXA, were comparable with those of male mice [42]. Additionally, despite consuming a low-fat diet, the OVX mice accumulated about $40 \%$ body fat and about a $25 \%$ reduction in percent body fat after undergoing a $30 \%$ calorie restriction [42]. Similarly, we found that OVX mice accumulated about $40 \%$ of body fat as assessed by MRI. Although naringenin reduced caloric intake by $\sim 14 \%$, total adiposity decreased by approximately $50 \%$. Additionally, naringenin significantly reduced perigonadal adipose tissue mass, even after controlling for body weight. Dietary naringenin has been linked to decreased adiposity in several male mouse studies independent of caloric intake $[12,34,35]$. Such data further suggest that the effect of naringenin on adiposity is not simply attributable to lower caloric intake.

Increased visceral fat and pro-inflammatory activity have been observed in postmenopausal women $[43,44]$. Previous studies indicate adipose tissues inflammation occurred early in OVX mice (e.g., 12 weeks after ovariectomy) and progressively worsens as indicated by increased infiltration and activation of immune cells and decreased insulin sensitivity $[16,17]$. Genetic deletion of MCP1 reduces body fat, increases glucose tolerance, and 
ameliorates adipose inflammation in visceral fat pad in OVX mice, but has no effect on sham-operated mice. These findings suggest that MCP1 may be a mediator of OVX-induced metabolic disturbances attributed to adipose tissue inflammation [45]. Yoshida et al. demonstrated that naringenin inhibited high-fat-diet induced toll-like receptor 2 mRNA expression and suppressed mRNA levels of proinflammatory mediators, TNF $\alpha$ and MCP1, in epididymal/perigonadal adipose tissue [35]. We found that naringenin down-regulated mRNA levels of MCP1 but not TNF $\alpha$ in perigonadal fat. Future studies will investigate the mechanisms of naringenin action on adipose tissue inflammation of OVX mice.

Interestingly, we found that naringenin significantly reduced MCP1 and IL6 mRNA in perigonadal adipose tissue, but had no significant effect on these markers of inflammation in subcutaneous adipose tissue. Rogers et al. [16] suggested that adipose tissue inflammation associated with OVX is more severe in perigonadal adipose tissue than in subcutaneous adipose tissue, which may explain why we found a more significant effect of naringenin on perigonadal adipose tissue inflammation. Additionally, by LC/MS-MS analyses, naringenin accumulated 1.7 fold more in perigonadal adipose than in subcutaneous adipose tissue after 11 weeks of $3 \%$ naringenin supplementation $(3.03 \pm 2.00 \mu$ mole $/ \mathrm{kg}$ vs. $5.11 \pm 1.26 \mu \mathrm{mole} / \mathrm{kg}$, data unpublished), suggesting that naringenin may have less influence on subcutaneous adipose tissue.

Estrogen deficiency has been connected to hepatic fat accumulation in women and rodents $[3,26]$. In the present study, we demonstrated that naringenin supplementation led to decreased hepatic lipid accumulation and changed mRNA levels of some genes involved in de novo lipogenesis and fatty acid oxidation. Several studies have shown that naringenin induces PPAR $\alpha$ activity and downstream enzymes involved in fatty acid oxidation, such as CPT $1 \alpha$, UCP2, and ACOX1 [11,13]. Interestingly, we observed increased Cpt $1 \alpha$ mRNA levels but decreased Acox1 levels, implicating discordance in mitochondrial and peroxisomal $\beta$-oxidation in response to OVX and naringenin treatment. Goldwasser et al. [14] also showed that naringenin induced a fasted-like state in hepatocytes, inhibiting fatty acid and cholesterol synthesis and increasing fatty acid oxidation. Consistent with these in vitro results, we also observed increased Srebf1 and PGC1 $\alpha$ mRNA in the liver of mice supplemented with naringenin. However, we did not observe increased expression microsomal triglyceride transfer protein (Mttp) expression and were unable to detect low-density lipoprotein (LDL) receptor mRNA expression (data not shown), which are downstream genes of Srebf1 and inducible by naringenin in vitro [46]. Additionally, we found increased hepatic expression of genes controlling gluconeogenesis in the mice fed naringenin, a normal physiological reaction in response to fasting. Lack of induction of these two genes in the $\mathrm{CON}$ mice may be related to their high levels of fasting glucose.

We demonstrated that naringenin decreased plasma cholesterol but did not change the levels of plasma triacylglyceride and non-esterified fatty acids in OVX mice. However, the effects of OVX on blood triacylglyceride and non-esterified fatty acids are mixed. Several studies have shown no difference in blood triacylglyceride and non-esterified fatty acids between sham-operated and OVX rodents [16,17,39,47-49], while others have observed increased levels in OVX rodents [27,50]. It is possible that OVX had no effect on triacylglyceride and non-esterified fatty acids in the current study. Therefore we did not observe changes in plasma triacylglyceride and non-esterified fatty acids in naringenin-supplemented mice.

Estrogen deficiency-associated metabolic disturbances have been widely demonstrated in OVX mice $[16,17,37$, $39,40,42,51]$. There were several limitations of the present study. Due to the atrophy of the uterus observed in our OVX mice, we could not measure the weight of uterus to determine whether there is an estrogenic or anti-estrogenic effect of naringenin. Additionally, we did not have shamoperated mice as a control to evaluate the degrees of metabolic disturbances in our OVX mice and thus the extent of naringenin effects. However, the elevated fasting glucose, adiposity, and hepatic lipid accumulation observed in OVX mice in the present study were markedly reduced with naringenin supplementation. In addition, as mentioned earlier, we did not design this study to include a pair-fed group since previous studies evaluating metabolic effects of diets with naringenin in male rodents did not observe differences in food intake.

Naringenin readily accumulates in plasma after ingestion of orange juice, grapefruit juice [52], and tomato paste or sauce [53], suggesting that it is bioavailable in individuals who consume naringenin food sources regularly. In the present study, we found that mice developed higher fasting glucose, adiposity, and hepatic steatosis after loss of ovarian function, similar to what has been observed in postmenopausal women $[3,31]$. Naringenin supplementation attenuated these estrogen-deficiency-associated metabolic disturbances in OVX female mice, suggesting the potential influence of dietary naringenin on metabolic syndrome in postmenopausal women. Further work in pre-clinical and human intervention studies will help to determine if naringenin is able to protect against menopausal-associated metabolic syndrome in humans.

\section{Additional file}

Additional file 1: Table S1. Composition of experimental diets. Table S2: Real-time PCR primers and probes of Taqman gene expression assay. Figure S1: Illustration of different fat depots analyzed in the present paper. Total, intra-abdominal, and subcutaneous adiposity were analyzed 
by MRI using a Bruker Biospin 94/30 magnet (Billerica, MA, USA) and a 70 $\mathrm{mm}$ diameter linear volume coil. T1-weighted coronal images of the whole mouse torso were collected using a respiratory-gated RARE sequence $\left(T R / T E=1570 / 7.5 \mathrm{~ms}\right.$, RARE factor $=4, F O V=70 \times 45 \mathrm{~mm}^{2}$, matrix size $=256 \times 192$, slice thickness $=1 \mathrm{~mm}$, navg $=2$ ). Mice were anesthetized with $2-2.5 \%$ isoflurane mixed with 1 liter per minute carbogen $\left(95 \% \mathrm{O}_{2}+\right.$ $5 \% \mathrm{CO}_{2}$ ) and maintained with $1-1.5 \%$ isoflurane. Physiologic parameters such as the electrocardiography, respiration and the temperature of the animals were monitored using a small animal monitoring system (Model 1025, Small Animals Instruments, Inc. Stony Brook, NY, USA). Otsu segmentation [1] was used to segment the mouse body from background. A connected components algorithm [2] was used to label the background objects in the image and 'fill' any holes in the segmented body image. The abdominal cavity was manually outlined in the images and a global threshold of 120 grey level intensity was chosen to segment fat from surrounding tissue. The whole body and abdominal masks were then used to calculate the percentage of segmented voxels in the whole body and abdomen, respectively. Subcutaneous fat was calculated by subtracting intra-abdominal fat from total fat. Figure S2: Insulin tolerance test was performed at week 21 to determine insulin sensitivity ( $C O N, n=4$; NAR $n=5)$. After a 5 h-fast, insulin was administered intraperitoneally at a dose of 0.4 U/kg body weight (Humulin R, Eli Lilly and Co., Indianapolis, IN, USA). Glucose was measured from tail vein blood using a OneTouch Ultra blood glucose meter immediately prior to insulin injection (time 0) and 15, 30, 45, 60,90 , and 120 minutes following the injection. The values were normalized to baseline (time 0 ) values and the percent change from baseline was plotted over time. The response curve was used to calculate area under the curve (AUC) [3]. Graphical representation of the calculated area under curve (AUC) between time point 0 to 120 min was shown on the top right.

\section{Abbreviations}

CON: Control diet group; HOMA-IR: Homeostasis model assessment of insulin resistance; NAR: Naringenin diet group; OVX: Ovariectomized;

PGAT: Perigonadal adipose tissue; SCAT: Subcutaneous adipose tissue.

\section{Competing interests}

The authors declare that they have no competing interests.

\section{Authors' contributions}

J-YK: contributed to the design of the study, performed the experiments, conducted data analysis and obtained external support for the work. KLK \& EMH \& RMC: assisted with experiments and tissue collection and contributed to data interpretation. KAP: conducted and analyzed MRI images. RRA: provided statistical modeling and analysis. SRS: helped with tissue collection and performed histological analysis. LDY: contributed to data interpretation. MAB: designed the study, obtained external support for the work, and oversaw data analysis and interpretation. All authors read, wrote sections of the manuscript, and approved the final manuscript.

\section{Acknowledgements}

The authors thank Josephine Fouts for assistance with mouse experimentation, and Dr. Santosh K. Maurya and Dr. Muthu Periasamy for support and assistance with indirect calorimetry measurements. The authors would like to acknowledge the OSU Small Animal Imaging Core for MR imaging. This research was supported by the Carol S. Kennedy Endowment (M.A.B.), NCl 1R03CA162551 (M.A.B.) and the Food Innovation Center Doctoral Research Grant (J-Y. K).

\section{Author details}

'Department of Human Sciences, Human Nutrition Program, College of Education and Human Ecology, The Ohio State University, Campbell Hall 302, 1787 Neil Avenue, Columbus, Ohio 43210, USA. ${ }^{2}$ Department of Dairy Science, Faculty of Agriculture, Cairo University, Giza, Egypt. ${ }^{3}$ Department of Biomedical Informatics, College of Medicine, The Ohio State University, Columbus, Ohio, USA. ${ }^{4}$ Division of Biostatistics, College of Public Health, The Ohio State University, Columbus, Ohio, USA. ${ }^{5}$ Department of Surgery, College of Medicine, The Ohio State University, Columbus, Ohio, USA.

Received: 22 September 2014 Accepted: 23 December 2014 Published: 13 January 2015

\section{References}

1. Davis SR, Castelo-Branco C, Chedraui P, Lumsden MA, Nappi RE, Shah D, et al. Understanding weight gain at menopause. Climacteric. 2012;15:419-29.

2. Kaaja RJ. Metabolic syndrome and the menopause. Menopause Int. 2008;14:21-5.

3. Suzuki A, Abdelmalek MF. Nonalcoholic fatty liver disease in women. Womens Health (Lond Engl). 2009;5:191-203.

4. Barrett-Connor E. Menopause, atherosclerosis, and coronary artery disease. Curr Opin Pharmacol. 2013;13:186-91.

5. Rossouw JE, Anderson GL, Prentice RL, LaCroix AZ, Kooperberg C, Stefanick $M L$, et al. Risks and benefits of estrogen plus progestin in healthy postmenopausal women: principal results From the Women's Health Initiative randomized controlled trial. JAMA. 2002;288:321-33.

6. Terao J. Dietary flavonoids as antioxidants. Forum Nutr. 2009;61:87-94.

7. Meydani M, Hasan ST. Dietary polyphenols and obesity. Nutrients. 2010:2:737-51.

8. Davies JN, Hobson GE. The constituents of tomato fruit-the influence of environment, nutrition, and genotype. Crit Rev Food Sci Nutr. 1981;15:205-80

9. Kawaii S, Tomono Y, Katase E, Ogawa K, Yano M. Quantitation of flavonoid constituents in citrus fruits. J Agric Food Chem. 1999;47:3565-71.

10. Purushotham A, Tian M, Belury MA. The citrus fruit flavonoid naringenin suppresses hepatic glucose production from Fao hepatoma cells. Mol Nutr Food Res. 2009;53:300-7.

11. Huong DT, Takahashi Y, Ide T. Activity and mRNA levels of enzymes involved in hepatic fatty acid oxidation in mice fed citrus flavonoids Nutrition. 2006;22:546-52.

12. Mulvihill EE, Allister EM, Sutherland BG, Telford DE, Sawyez CG, Edwards JY, et al. Naringenin prevents dyslipidemia, apolipoprotein B overproduction, and hyperinsulinemia in LDL receptor-null mice with diet-induced insulin resistance. Diabetes. 2009;58:2198-210.

13. Cho KW, Kim YO, Andrade JE, Burgess JR, Kim YC. Dietary naringenin increases hepatic peroxisome proliferators-activated receptor alpha protein expression and decreases plasma triglyceride and adiposity in rats. Eur J Nutr. 2011;50:81-8.

14. Goldwasser J, Cohen PY, Yang E, Balaguer P, Yarmush ML, Nahmias Y. Transcriptional regulation of human and rat hepatic lipid metabolism by the grapefruit flavonoid naringenin: role of PPARalpha. PPARgamma and LXRalpha PLoS One. 2010;5:e12399.

15. Alam MA, Subhan N, Rahman MM, Uddin SJ, Reza HM, Sarker SD. Effect of citrus flavonoids, naringin and naringenin, on metabolic syndrome and their mechanisms of action. Adv Nutr. 2014;5:404-17.

16. Rogers NH, Perfield 2nd JW, Strissel KJ, Obin MS, Greenberg AS. Reduced energy expenditure and increased inflammation are early events in the development of ovariectomy-induced obesity. Endocrinology. 2009;150:2161-8

17. Vieira Potter VJ, Strissel KJ, Xie C, Chang E, Bennett G, Defuria J, et al. Adipose tissue inflammation and reduced insulin sensitivity in ovariectomized mice occurs in the absence of increased adiposity. Endocrinology. 2012;153:4266-77.

18. Peters J. Animal and Human Calorimetry - Mclean, Ja, Tobin, C. American Journal of Physical Anthropology. 1989;78:127-127.

19. Folch J, Lees M, Sloane Stanley GH. A simple method for the isolation and purification of total lipides from animal tissues. J Biol Chem. 1957;226:497-509.

20. Pacheco YM, Perez-Camino MC, Cert A, Montero E, Ruiz-Gutierrez V. Determination of the molecular species composition of diacylglycerols in human adipose tissue by solid-phase extraction and gas chromatography on a polar phase. J Chromatogr B Biomed Sci Appl. 1998;714:127-32.

21. Danno H, Jincho Y, Budiyanto S, Furukawa Y, Kimura S. A simple enzymatic quantitative analysis of triglycerides in tissues. J Nutr Sci Vitaminol (Tokyo). 1992;38:517-21.

22. Wendel AA, Purushotham A, Liu LF, Belury MA. Conjugated linoleic acid fails to worsen insulin resistance but induces hepatic steatosis in the presence of leptin in ob/ob mice. J Lipid Res. 2008;49:98-106.

23. Livak KJ, Schmittgen TD. Analysis of relative gene expression data using real-time quantitative PCR and the 2(-Delta Delta C(T)) Method. Methods. 2001;25:402-8.

24. Tschop MH, Speakman JR, Arch JR, Auwerx J, Bruning JC, Chan L, et al. A guide to analysis of mouse energy metabolism. Nat Methods. 2012;9:57-63.

25. Ryan AS, Elahi D. The effects of acute hyperglycemia and hyperinsulinemia on plasma leptin levels: its relationships with body fat, visceral adiposity, and age in women. J Clin Endocrinol Metab. 1996;81:4433-8. 
26. Lavoie JM, Pighon A. NAFLD, Estrogens, and Physical Exercise: The Animal Model. J Nutr Metab. 2012;2012:914938.

27. Leite RD, Prestes J, Bernardes CF, Shiguemoto GE, Pereira GB, Duarte JO, et al. Effects of ovariectomy and resistance training on lipid content in skeletal muscle, liver, and heart; fat depots; and lipid profile. Appl Physiol Nutr Metab. 2009;34:1079-86

28. Jou J, Choi SS, Diehl AM. Mechanisms of disease progression in nonalcoholic fatty liver disease. Semin Liver Dis. 2008:28:370-9.

29. Kallwitz ER, McLachlan A, Cotler SJ. Role of peroxisome proliferatorsactivated receptors in the pathogenesis and treatment of nonalcoholic fatty liver disease. World J Gastroenterol. 2008;14:22-8.

30. Xue F, Michels KB. Diabetes, metabolic syndrome, and breast cancer: a review of the current evidence. Am J Clin Nutr. 2007;86:8823-835.

31. Carr MC. The emergence of the metabolic syndrome with menopause. J Clin Endocrinol Metab. 2003;88:2404-11.

32. Assini JM, Mulvihill EE, Sutherland BG, Telford DE, Sawyez CG, Felder SL, et al. Naringenin prevents cholesterol-induced systemic inflammation, metabolic dysregulation, and atherosclerosis in Ldlr(-)/(-) mice. J Lipid Res. 2013;54:711-24

33. Kannappan S, Anuradha CV. Naringenin enhances insulin-stimulated tyrosine phosphorylation and improves the cellular actions of insulin in a dietary model of metabolic syndrome. Eur J Nutr. 2010;49:101-9.

34. Mulvihill EE, Assini JM, Sutherland BG, DiMattia AS, Khami M, Koppes JB, et al. Naringenin decreases progression of atherosclerosis by improving dyslipidemia in high-fat-fed low-density lipoprotein receptor-null mice. Arterioscler Thromb Vasc Biol. 2010;30:742-8.

35. Yoshida H, Watanabe W, Oomagari H, Tsuruta E, Shida M, Kurokawa M. Citrus flavonoid naringenin inhibits TLR2 expression in adipocytes. J Nutr Biochem. 2013;24:1276-84.

36. Brown LM, Clegg DJ. Central effects of estradiol in the regulation of food intake, body weight, and adiposity. J Steroid Biochem Mol Biol. 2010;122:65-73.

37. Witte MM, Resuehr D, Chandler AR, Mehle AK, Overton JM. Female mice and rats exhibit species-specific metabolic and behavioral responses to ovariectomy. Gen Comp Endocrinol. 2010;166:520-8.

38. D'Eon TM, Souza SC, Aronovitz M, Obin MS, Fried SK, Greenberg AS. Estrogen regulation of adiposity and fuel partitioning. Evidence of genomic and non-genomic regulation of lipogenic and oxidative pathways. J Biol Chem. 2005;280:35983-91.

39. Ludgero-Correia Jr A, Aguila MB, Mandarim-de-Lacerda CA, Faria TS. Effects of high-fat diet on plasma lipids, adiposity, and inflammatory markers in ovariectomized C57BL/6 mice. Nutrition. 2012;28:316-23.

40. Lovejoy JC, Champagne CM, de Jonge L, Xie H, Smith SR. Increased visceral fat and decreased energy expenditure during the menopausal transition. Int J Obes (Lond). 2008;32:949-58.

41. Sowers M, Zheng H, Tomey K, Karvonen-Gutierrez C, Jannausch M, Li X, et al. Changes in body composition in women over six years at midlife: ovarian and chronological aging. J Clin Endocrinol Metab. 2007;92:895-901.

42. Hong J, Stubbins RE, Smith RR, Harvey AE, Nunez NP. Differential susceptibility to obesity between male, female and ovariectomized female mice. Nutr J. 2009;8:11.

43. Pfeilschifter J, Koditz R, Pfohl M, Schatz H. Changes in proinflammatory cytokine activity after menopause. Endocr Rev. 2002;23:90-119.

44. Lee CG, Carr MC, Murdoch SJ, Mitchell E, Woods NF, Wener MH, et al. Adipokines, inflammation, and visceral adiposity across the menopausal transition: a prospective study. J Clin Endocrinol Metab. 2009;94:1104-10.

45. Kim WK, Choi EK, Sul OJ, Park YK, Kim ES, Yu R, et al. Monocyte chemoattractant protein-1 deficiency attenuates oxidative stress and protects against ovariectomy-induced chronic inflammation in mice. PLoS One. 2013;8:e72108.

46. Borradaile NM, de Dreu LE, Huff MW. Inhibition of net HepG2 cell apolipoprotein B secretion by the citrus flavonoid naringenin involves activation of phosphatidylinositol 3-kinase, independent of insulin receptor substrate-1 phosphorylation. Diabetes. 2003;52:2554-61.

47. Paquette A, Shinoda M, Rabasa Lhoret R, Prud'homme D, Lavoie JM. Time course of liver lipid infiltration in ovariectomized rats: impact of a high-fat diet. Maturitas. 2007;58:182-90.

48. Paquette A, Wang D, Jankowski M, Gutkowska J, Lavoie JM. Effects of ovariectomy on PPAR alpha, SREBP-1C, and SCD-1 gene expression in the rat liver. Menopause. 2008;15:1169-75.
49. Wohlers $L M$, Jackson $K C$, Spangenburg EE. Lipolytic signaling in response to acute exercise is altered in female mice following ovariectomy. J Cell Biochem. 2011;112:3675-84.

50. Wohlers LM, Spangenburg EE. 17beta-estradiol supplementation attenuates ovariectomy-induced increases in ATGL signaling and reduced perilipin expression in visceral adipose tissue. J Cell Biochem. 2010;110:420-7.

51. Stubbins RE, Najjar K, Holcomb VB, Hong J, Nunez NP. Oestrogen alters adipocyte biology and protects female mice from adipocyte inflammation and insulin resistance. Diabetes Obes Metab. 2012;14:58-66.

52. Erlund I, Meririnne E, Alfthan G, Aro A. Plasma kinetics and urinary excretion of the flavanones naringenin and hesperetin in humans after ingestion of orange juice and grapefruit juice. J Nutr. 2001;131:235-41.

53. Bugianesi R, Catasta G, Spigno P, D'Uva A, Maiani G. Naringenin from cooked tomato paste is bioavailable in men. J Nutr. 2002;132:3349-52.

doi:10.1186/1743-7075-12-1

Cite this article as: Ke et al: The flavonoid, naringenin, decreases adipose tissue mass and attenuates ovariectomy-associated metabolic disturbances in mice. Nutrition \& Metabolism 2015 12:1.

\section{Submit your next manuscript to BioMed Central and take full advantage of:}

- Convenient online submission

- Thorough peer review

- No space constraints or color figure charges

- Immediate publication on acceptance

- Inclusion in PubMed, CAS, Scopus and Google Scholar

- Research which is freely available for redistribution

Submit your manuscript at www.biomedcentral.com/submit
C BioMed Central 